\title{
The Holonomy of the Determinant of Cohomology of an Algebraic Bundle
}

\author{
H. Gillet ${ }^{1}$ and C. Soulé ${ }^{2}$ \\ ${ }^{1}$ Department of Mathematics, University of Illinois at Chicago, \\ Box 4348, Chicago, Il 60680, USA \\ ${ }^{2}$ I.H.E.S., 35, Route de Chartres, F-91440 Bures-sur-Yvette, France
}

\begin{abstract}
The purpose of this note is to remark that Theorem 3.7 in [1], when combined with the work of Bismut and Freed [2], leads, in the algebraic case, to an improvement of both results concerning the holonomy of determinant line bundles.
\end{abstract}

So let $f: X \rightarrow Y$ be a smooth proper map between projective complex manifolds. Choose a metric $h$ on the relative tangent space $T_{X / Y}$ and a smooth complement $T^{H} X$ to $T_{X / Y}$ in $T X$. We assume that $\left(f, T^{H} X, h\right)$ is a Kähler fibration in the sense of [3], i.e. there exists a closed $(1,1)$ form $\omega$ on $X$ for which $T_{X / Y}$ and $T^{H} X$ are orthogonal, and $\omega$ restricts to the $(1,1)$ form associated to $h$ on $T_{X / Y}$.

Let $E$ be an algebraic vector bundle on $X$, endowed with a smooth Hermitian metric $h_{E}$. The (algebraic) determinant line bundle

$$
\lambda(E)=\operatorname{det} R f_{*}(E)
$$

may then be equipped with its Quillen metric [3], whose associated connection we denote by $\nabla_{Q}$.

Given a smooth loop

$$
\gamma: S^{1} \rightarrow Y
$$

we want to compute the holonomy of $\nabla_{Q}$ along $\gamma$. By pulling back $f$ along $\gamma$ we get a commutative diagram of real manifolds,

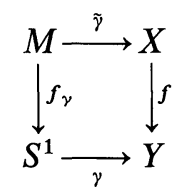

with $T M \cong \tilde{\gamma}^{*}\left(T_{X / Y}\right) \oplus f_{\gamma}^{*}\left(T S^{1}\right)$ (because of the choice of $\left.T^{H} X\right)$. Endow $T M$ with the orthonormal direct sum of $\tilde{\gamma}^{*}(h)$ with the metric on $T S^{1}$ giving norm one to $\frac{d}{d t}$ and invariant by rotation. Let $D$ be the Dirac operator acting on the sections of 
$E \otimes F \otimes \xi^{-1}$ on $M$, where $F$ is the (locally defined) bundle of spinors and $\xi$ a square root of $\operatorname{det}\left(T_{X / Y}^{*(0,1)}\right)$. Denote by $\eta(0)$ the $\eta$-invariant of $D$, by $h(D)$ the dimension of the kernel of $D$, and by $\chi$ the Euler characteristic of $E$ on any fiber of $f$ on $\gamma\left(S^{1}\right)$.

Theorem. The holonomy $\mu(\gamma)$ of $\nabla_{Q}$ around $\gamma$ is

$$
\mu(\gamma)=(-1)^{\chi} \exp (2 \pi i(\eta(0)+h(D)) / 2) \in S^{1} .
$$

To prove this theorem, for every real number $\varepsilon>0$, we define $D^{\varepsilon}$ as $D$ above, except that $\frac{d}{d t}$ has norm $\varepsilon^{-1}$ (so $D=D^{1}$ ). If $\eta^{\varepsilon}(0)$ is the êta invariant of $D^{\varepsilon}$, and $\bar{\eta}^{\varepsilon}(0)$ $=\left(\eta^{\varepsilon}(0)+\operatorname{dim}\left(D^{\varepsilon}\right) / 2\right)$, we know from [2] that

$$
\lim _{\varepsilon \rightarrow 0}(-1)^{\chi} \exp \left(2 \pi i \bar{\eta}^{\varepsilon}(0)\right)=\mu(\gamma) \text {. }
$$

On the other hand, the proof of Theorem 3.7 in [1] shows that, for any $\varepsilon>0$,

$$
\mu(\gamma) \equiv \exp \left(2 \pi i \bar{\eta}^{\varepsilon}(0)\right)
$$

is $S^{1} \otimes \mathbb{Q}$. Since we know from [2] that $\bar{\eta}^{\varepsilon}(0)$ is a continuous function of $\varepsilon$, the number $\exp \left(2 \pi i \bar{\eta}^{\varepsilon}(0)\right) \in S^{1}$ depends continuously on $\varepsilon$. It is constant modulo roots of unity, therefore it is constant. We conclude that

$$
\mu(\gamma)=\lim _{\varepsilon \rightarrow 0}(-1)^{\chi} \exp \left(2 \pi i \bar{\eta}^{\varepsilon}(0)\right)=(-1)^{\chi} \exp \left(2 \pi i \bar{\eta}^{\varepsilon}(0)\right)
$$

for all $\varepsilon$, in particular for $\varepsilon=1$. In other words, the adiabatic limit of [2] is stationary under our hypotheses.

\section{References}

1. Gillet, H., Soulé, C.: Arithmetic Chow groups and differential characters. In: Algebraic $K$-theory: connections with geometry and topology. Jardine, J.F., Snaith, V.P. (eds.), pp. 30-68. Dordrecht: Kluwer Academic 1989

2. Bismut, J.-M., Freed, D.-S.: The analysis of elliptic families. II. Dirac operators, êta invariants and the holonomy theorem. Commun. Math. Phys. 107, 103-163 (1986)

3. Bismut, J.-M., Gillet, H., Soulé, C.: Analytic torsion and holomorphic determinant bundles. I-III. Commun. Math. Phys. 115, 49-78, 78-126, 301-351 (1988)

Communicated by A. Jaffe

Received December 1, 1989 